\title{
DETERMINACIÓN DE LA SEROPREVALENCIA DE CISTICERCOSIS PORCINA E IDENTIFICACIÓN DE TENIASIS HUMANA EN PERSONAS CRIADORAS DE CERDOS EN EL ÁREA URBANA DEL MUNICIPIO DE COYAIMA TOLIMA
}

\author{
JULIO CÉSAR GIRALDO FORERO ${ }^{1}$ MILENA MARCELA RIAÑO RODRÍGUEZ ${ }^{2}$ \\ LUIS REINEL VÁSQUEZ ARTEAGA ${ }^{3}$ \\ ${ }^{1} M . S c$. Esp. Docente Investigador, \\ líder Grupo de Investigación en Parasitología y Microbiología Tropical (GIPAMT). \\ Facultad de Ingeniería, Administración y Ciencias Básicas. \\ Programa de Biología. Universidad INCCA de Colombia. \\ Bogotá, Colombia \\ ${ }^{2}$ Bióloga. Universidad INCCA de Colombia. Bogotá, Colombia \\ ${ }^{3}$ M.Sc. Esp. Docente Investigador, líder Grupo Centro de Estudios en Microbiología y Parasitología (CEMPA) \\ Facultad de Ciencias de la Salud, Departamento de Medicina Interna. \\ Universidad del Cauca. Popayán, Cauca, Colombia \\ ${ }^{1}$ Correspondencia: Julio Cesar Giraldo. jcesargiraldo@gmail.com \\ Recibido: 7 Diciembre 2016 Aceptado: 3 Abril 2017
}

\begin{abstract}
RESUMEN
Introducción: La cisticercosis porcina causada por la etapa larval de Taenia solium, ocasiona graves pérdidas económicas para sus tenedores y un riesgo para la salud pública en Colombia y en el mundo especialmente en Asia, África y otros países de Latino América. Esta parasitosis se propaga con facilidad en zonas con condiciones higiénicas sanitarias, ambientales y socio económicas precarias.

Objetivo: Determinar la seroprevalencia de cisticercosis porcina empleando el ensayo inmunoenzimático (ELISA) y la detección de teniasis humana por examen coproparasitológico en personas criadoras de cerdos en el área urbana.

Materiales y Métodos: Se tomó muestra de sangre a 102 porcinos por punción en el pabellón auricular y se impregnaron en discos de papel de filtro para detectar anticuerpos anticisticerco empleando la técnica de ELISA con la fracción antigénica de $53 \mathrm{kDa}$. A los tenedores de porcinos y demás participantes conformado por un grupo de 159 personas, se les solicitó una muestra de heces, que fue conservada en reactivo de Mertiolate Yodo Formol al $5 \%$ (MIF). Se preservó a $4^{\circ} \mathrm{C}$, para su procesamiento se empleó la técnica de concentración de formol-éter, el diagnóstico se realizó por observación microscópica con objetivo de 40X.

Resultados: De los 102 porcinos, el 17\% (17/102) presentaron anticuerpos anticisticerco. Con respecto a las variables sociodemográficas del estudio se observó mayor asociación con fuentes de agua, pacientes teniásicos, condiciones ambientales, tipo de alimentación. La prevalencia general de parasitismo intestinal en los criadores fue de $43.3 \%$, y en el 1.9\% (3/159) se hallaron huevos de Taenia spp, Se identificó a las fuentes de agua y la tenencia de porcinos como variable estadísticamente significativa $(\mathrm{p} \leq 0,005)$ con la presencia de parasitismo intestinal en la comunidad.
\end{abstract}

Conclusión: El estudio reveló un valor significativo de seroprevalencia de cisticercosis porcina en el área evaluada, ocasionando un serio problema de salud pública en la comunidad coyaimuna.

Palabras clave: Taenia solium; Cisticercosis Porcina; Parasitismo Intestinal; prueba ELISA. 


\title{
DETERMINATION OF SEROPREVALENCE OF SWINE CYSTICERCOSIS AND IDENTIFICATION OF HUMAN TENIASIS IN PIG CREATING PERSONS IN THE URBAN AREA SOF THE COYAIMA TOLIMA MUNICIPALITY
}

\begin{abstract}
Introduction: Swine cysticercosis caused by the larval stage of Taenia solium, causes serious economic losses for their holders and a risk to public health in Colombia and the world, especially in Asia, Africa and other Latin American countries. This parasitic disease is spread easily in areas with hygienic sanitary, environmental and socio economic precarious.

Objective: determine the seroprevalence of cysticercosis swine using the essay Enzyme Immunoassay (ELISA) and the detection of taeniasis human by test coproparasitologic in people brooders of pigs in the area urban.

Materials and methods: Took blood sample to swine 102 puncture the pinna and they impregnated filter paper disks to detect Flisser antibodies using the ELISA technique with $53 \mathrm{kDa}$ antigenic fraction. To them holders of pigs and others participating conformed by a group of 159 people, is les requested a shows of Lee that was preserved in reagent of Mertiolate iodine formalin to the $5 \%$ (MIF). Is preserved to $4{ }^{\circ} \mathrm{C}$, for its processing is employed the technical of concentration of formol-eter, the diagnosis is made by observation microscopic with objective of $40 \mathrm{X}$.

Results: Of the 102 pigs, the 17\% (17 / 102) presented antibodies Flisser. With respect to the variable sociodemographic of the study is observed greater association with sources of water, patient's teniasicos, conditions environmental, type of power. The general prevalence of intestinal parasitism in the breeders was $43.3 \%$ and $1.9 \%$ (3/159) found eggs of Taenia spp., identified the sources of water and holding of pigs as variables statistically significant $(p \leq 0$. 005) with the presence of intestinal parasitism in the community.
\end{abstract}

Conclusion: The study revealed a significant value of seroprevalence of porcine cysticercosis in the evaluated area, causing a serious problem of public health in the community coyaimuna.

Keywords: Taenia solium; Cysticercosis swine; Parasitism Intestinal; Test ELISA. 


\section{DETERMINAÇÃO DA SEROPREVALÊNCIA DA CÍTICA E SUA IDENTIFICAÇÃO DA TENIASE HUMANA NO PIG CRIANDO PESSOAS NA ÁREA URBANA DO MUNICÍPIO DE COYAIMA TOLIMA}

\section{RESUMO}

Introdução: Cisticercose porcina causada pelo estágio larval de Taenia solium, provoca sérias perdas econômicas para os seus titulares e um risco para a saúde pública na Colômbia e no mundo, especialmente na Ásia, África e outros países da América Latina. Esta doença parasitária é disseminada facilmente em áreas com higiene higiênica, ambiental e socioeconômica precária.

Objetivo: Determinar a soroprevalência de cisticercose porco utilizando o ensaio imunoenzimático (ELISA) e a deteç̧ão de taeníase humana por teste coproparasitológico em pessoas criadas por porcos na área urbana.

Materiais e métodos: Tomou amostras de sangue para suínos 102 perfurando o pinna e impregnaram discos de papel de filtro para detectar anticorpos Flisser usando a técnica ELISA com fração antigênica de 53kDa. A eles, titulares de porcos e outros participantes, conformados por um grupo de 159 pessoas, solicita-se um show de Lee que foi preservado em reagente de Mertiolate iodine formalin a $5 \%$ (MIF). É preservada a $4{ }^{\circ} \mathrm{C}$, para o processamento é empregada a técnica de concentração de formol eter, o diagnóstico é feito por observação microscópica com objetivo de $40 \mathrm{X}$.

Resultados: Dos 102 porcos, 17\% (17/102) apresentaram anticorpos Flisser. Com relação à variável sociodemográfica do estudo, observa-se maior associação com fontes de água, teniasicos do paciente, condições ambientais, tipo de poder. A prevalência geral de parasitismo intestinal nos criadores foi de 43,3\% e 1,9\% (3/159) encontraram ovos de Taenia spp., Identificaram as fontes de água e a retenção de porcos como variáveis estatisticamente significativas $(p \leq 0.005)$ com a presença De parasitismo intestinal na comunidade.

Conclusões: O estudo revelou um valor significativo de seroprevalência de cisticercose porcina na área avaliada, causando um grave problema de saúde pública na comunidade coyaimuna.

Palavras-chave: Taenia solium; Cisticercose suina; Parasitism Intestinal; Teste ELISA 


\section{Introducción}

El complejo teniosis/cisticercosis (TC) es una zoonosis desatendida causado por el céstodo Taenia solium, es considerado un problema de salud pública en países de América Latina, África y Asia. En países en desarrollo este complejo parasitario se establece debido a las deficientes condiciones sociales, económicas, culturales y de crianza no tecnificada de cerdos en zonas endémicas (1-3).

El ser humano es el hospedero definitivo donde se encuentra la forma adulta de $T$. solium (tenia o lombriz solitaria, términos en Colombia), eventualmente de forma accidental éste y el cerdo se infectan al consumir huevos del parásito adulto y desarrollan la cisticercosis (estadio larval) (4-6).

A partir de 1993, la cisticercosis fue declarada una zoonosis potencialmente erradicable por la International Task Force for Disease Erradication, tomando en consideración que la forma adulta solamente utiliza como hospedero definitivo al humano, el cual constituye la única fuente de huevos de T. solium. Se plantea la aplicación prioritaria de medidas de control a los porcinos, las cuales serían particularmente efectivas debido a que no hay reservorios silvestres y tampoco disponibilidad de productos farmacológicos eficaces (7-10).

Según las cifras de la Organización Mundial de la Salud (OMS), más del $80 \%$ de los 50 millones de personas afectadas en el mundo por epilepsia, viven en países de ingresos bajos. Siendo la larva de T. solium, la causa del $30 \%$ de los casos de epilepsia en muchas zonas endémicas donde los humanos tienen convivencia con los cerdos (11-14). En China se han reportado cerca de 1.26 millones de portadores de la fase adulta y con cisticercosis tres millones (15). Con respecto al Norte de India se ha revelado una seroprevalencia general, en la zona urbana de $3,48 \%$ y rural de $4,64 \%$ (16). Sin embago, se encontró mayor prevalencia de cisticercosis porcina en América Latina en Guatemala, Honduras, México y Perú con un $17 \%(11,17-19)$.

La dispersión de la enfermedad es amplia en nuestro país, como lo indica el estudio realizado en 23 departamentos de Colombia en el periodo 2008-2010; quien reportó una seroprevalencia general en humanos de $8.55 \%$, el departamento con mayor frecuencia de anticuerpos fue Vaupés $40.19 \%$ y la menor Caldas $0.53 \%$, citando algunas de las prácticas que favorecen una infección por este parásito: la crianza de cerdos en con- diciones inadecuadas proporciona la infección de estos animales debido al contacto con heces humanas cuando no son eliminadas correctamente; de igual forma el estudio mostró que el $11,40 \%$ de la población seropositiva convive con animales dentro de la vivienda con número que osciló alrededor de seis cerdos. De modo similar, hábitos de la población como el no lavado de verduras antes de consumir, el sacrificio y la venta de carne sin control sanitario, el manejo y costumbre de consumo en la vía pública y la falta de control sanitario de la misma, al igual que el consumo con escasos procesos de cocción o cruda $(20,21)$.

Los reportes de diferentes autores en Colombia sobre la cisticercosis porcina, han registrado la prevalencia de esta parasitosis en diferentes zonas del departamento del Tolima, por el método de ELISA oscilando del $15.63 \%$ al $37.5 \%$ de casos positivos la prevalencia registrada en 1992 en Coyaima alcanzó 25,64\% (22), al igual que las investigaciones realizadas en los municipios de Moñitos y Los Córdobas (Córdoba) se evidenció una seroprevalencia para del 13,33\% y la presencia de cisticercos mediante observación post-mortén en 180 animales. Otros estudios realizados entre ellos el de dos comunidades rurales de Antioquia detectó una prevalencia de cisticercosis humana y porcina de $2,23 \%$ y $6,82 \%$ respectivamente, Pascuita del 1,17\% y Guacharaquero 2,23\%, Andagoya en el 2009 seroprevalencia en criadores y familiares del 8,7 \% y en los animales del 20,9 \% (23), lo que permite evidenciar que en Colombia ninguna zona es libre de cisticercosis debido a las malas prácticas pecuarias (20).

Diferentes técnicas de diagnóstico han sido descritas para detectar anticuerpos contra $T$. solium, en humanos y cerdos, como la palpación de la lengua en el caso de los cerdos, es especifica pero con baja sensibilidad. Dentro de las técnicas de laboratorio, se encuentra la prueba de fijación de complemento, hemaglutinación, radioinmunoensayo, (ELISA), técnicas de inmunotransferencia, Inmunoblot, Reacción en cadena de la polimerasa (PCR, por sus siglas en ingles), entre otras $(24,25)$. La prueba más específica desarrollada es la Inmunoelectrotransferencia blot ligado a enzimas (EITB), que tiene una especificidad de $100 \%$ y una sensibilidad que oscila entre 70 y $90 \%$ (26). Sin embargo, otros estudios han revelado una sensibilidad del $28 \%$ para casos individuales con quistes en el cerebro (27). El ensayo EITB ha sido ampliamente utilizado para el diagnóstico de la cisticercosis en muestras de suero humano y de cerdo, pero en países 
en desarrollo, la técnica ELISA es más utilizada por su mayor disponibilidad, sensibilidad y menor costo en comparación con Inmunoblot (24), en cuanto a la PCR se ha demostrado que esta presenta alta especificidad pero baja sensibilidad (28).

La recolección de sangre total en papel de filtro (PF) se ha utilizado durante más de cinco décadas (29), ya que ha demostrado entre las diferentes técnicas de serodiagnóstico ser un método que abarca diversas ventajas como: factibilidad de usarse en campo, fácil transporte y conservación (30), siendo ampliamente utilizada en varias partes del mundo para el diagnóstico de enfermedades infecciosas en diversas especies de animales $(25,31)$, este método tiene más aceptación que las extracciones convencionales de sangre. La colecta de sangre total en PF para los ensayos de anticuerpos tiene ventajas sobre el uso de muestras de suero: los requerimientos de equipos son mínimos, las lancetas estériles son baratas y el PF sustituye a las jeringas, tubos, centrífugas y refrigeradores que se requieren para las muestras de suero.

En cuanto al analisis coproparasitológico por microscopía óptica, busca la presencia de formas evolutivas móviles de parásitos de tamaño microscópico (trofozoítos, quistes de protozoos: Entamoeba histolytica, Giardia duodenalis, Neobalantidium coli, entre otros) así como larvas o huevos de helmintos: Strongyloides stercoralis, Ancylostoma duodenale, Necator americanus, Trichostrongylus spp, Paragonimus spp, Fasciola spp. Una de las técnicas mas utilizadas es la de formol éter, la cual sobresale del método de Beawer modificado, al detectar como positivas muestras adicionales, que en un solo coprológico directo habrían dado negativas para el diagnóstico de geohelmintos (32-36) grupo en el que se encuentran Ascaris lumbricoides, Trichuris trichiura, Strongyloides stercoralis. Sin embargo, la Ley 100 de 1993, autoriza para el sistema general de seguridad social coprológico directo para el diagnóstico de parasitosis intestinales y no técnicas de concentración como la de formol éter.

Este estudio se realizó con el objetivo de determinar la prevalencia de cisticercosis porcina mediante la técnica de ELISA utilizando la fracción de 53kDa como antígeno y establecer una asociación con variables sociodemográficas, como también la identificación de huevos de T. solium, por examen coproparasitológico en la población criadora de cerdos de la zona urbana del municipio de Coyaima (Tolima).

\section{Metodología}

Se realizó una investigación descriptiva de corte transversal para conocer el comportamiento del complejo teniasis/cisticercosis y las variables asociadas en la zona urbana del municipio de Coyaima (Tolima).

\section{Área y población de estudio}

El municipio de Coyaima (Tolima) en el área urbana tiene como característica la cría de cerdos, entre otras actividades y cuenta con un área territorial de $664.33 \mathrm{~km}^{2}$, de los cuales $658,13 \mathrm{~km}^{2}$ corresponden al sector rural (99\%) y $6,20 \mathrm{~km}^{2}$ al sector urbano (1\%); se encuentra a $392 \mathrm{msnm}$, con una temperatura promedio de $27^{\circ} \mathrm{C}$ (37). Coyaima tiene disponibilidad de agua superficial por el aporte regional de los ríos Saldaña y Magdalena que lo bordean, pero no posee agua potable por carencia de una planta de tratamiento (38). El número de habitantes reportados por el DANE hasta el 2010 es de 32.300 en total con la población del área urbana y rural (39). En cuanto al sector porcícola, un informe realizado por el Registro Mercantil en el 2013 manifiesta producción no tecnificada y de consumo local (40). La prestación de servicio de salud humana, está a cargo de un hospital de primer nivel y el animal por dos centros veterinarios. El sacrificio porcino se realiza domésticamente.

\section{Recolección de los datos}

El estudio se realizó entre los años en 2012 y 2013, la primera fase incluyó charlas informativas a la comunidad y provisión de material didáctico sobre el parasitismo intestinal, enfatizándose el ciclo epidemiológico de la cisticercosis porcina y humana, como los factores asociados al mismo y las consecuencias de este a corto, mediano y largo plazo. Mediante visita domiciliaria se obtuvo la firma de un consentimiento informado por los participantes y el diligenciamiento de una encuesta epidemiológica estructurada, que indagó sobre variables sociodemográficas (Tabla 1)

\section{Toma de muestras}

Se recolectaron102 muestras serológicas de porcinos por conveniencia realizando punción del pabellón auricular con lancetas estériles y absorción en discos de PF Whattman No 1 de $4 \mathrm{~mm}$ de diámetro los que se dispensaron en tubos Eppendorf estériles debidamente rotulados a los cuales se les adicionó $1 \mathrm{ml}$ de solución salina estéril y se almacenaron a $4^{\circ} \mathrm{C}$ para su elución. 
Se solicitó la recolección de una muestra de materia fecal a las 159 personas participantes en un recipiente estéril las cuales fueron rotuladas y preservadas con $30 \mathrm{ml}$ de reactivo de MIF al 5\%, se mezclaron hasta que su textura fue semiliquida se conservaron a $4^{\circ} \mathrm{C}$ y transportaron al laboratorio para su procesamiento.

\section{Adecuación de la prueba}

Las muestras fueron procesadas por la técnica ELISA indirecta, la cual utilizó como antígeno la fracción proteica de $53 \mathrm{kDa}$, obtenida por elución a partir de geles SDS- PEAGE al $10 \%$ del perfil electroforético de un extracto total, delipidado y sonicado de cisticercos vesiculares (41).

\section{Sueros controles}

Sueros positivos: se utilizaron los procedentes de porcinos en los que por palpación sublingual premorten se detectaron estadios vesiculares de $T$. solium, y en disección posmortem se extrajeron cisticercos tisulares, de igual manera las muestras de sangre total y suero se colectaron en discos de PF Wattman $\mathrm{N}^{\circ} 1 \mathrm{de} 4 \mathrm{~mm}$ de diámetro, las que se evaluaron por la técnica de ELISA. En las tres valoraciones, se halló presencia de anticuerpos para el metacéstodo de $T$. solium.

Sueros negativos: se emplearon los de animales a los cuales se les evaluó premortem por palpación sublingual, posmortem con cortes específicos en músculos linguales, bricep, tricep e intercostales, entre otros. La valoración serológica incluyó colecta de sangre total y suero en discos de PF, de igual manera que para el caso de los sueros positivos, la prueba de ELISA fue la técnica de referencia. Las muestras fueron negativas para la totalidad de las valoraciones.

\section{Condiciones de la Prueba de ELISA}

Se realizó la adición de 100 1 le buffer de cobertura por pozo en la placa con la fracción de $53 \mathrm{kDa}$ en concentración de $0.4 \mu \mathrm{g} / \mathrm{ml}$, se incubó a $4^{\circ} \mathrm{C}$ durante toda la noche. Se realizaron tres lavados cada uno de cinco minutos con buffer PBS pH 7.4-Tween 20 al $0.1 \%$, se adicionó por pozo $200 \mu l$ de solución de bloqueo con albúmina sérica bovina fracción $\mathrm{V}$ marca SIGMA al $0.1 \%$, posteriormente se incubó en cámara húmeda a $37^{\circ} \mathrm{C}$ durante una hora, se repitió el proceso de lavado anterior. Los sueros controles positivos y negativos se diluyeron en PBS pH 7.4, en proporción $1 / 1$. Se incubó nuevamente a $37^{\circ} \mathrm{C}$ durante una hora, se repitió el proceso de lavado descrito previamente. A continuación, se agregó $100 \mu l$ por pozo del conjugado
Anti-IgG porcina ligada a la enzima peroxidasa en dilución de 1/2500 en buffer PBS pH 7.4.; se incubó una hora a $37^{\circ} \mathrm{C}$. Se reveló la reacción Ag-Ac, con $200 \mu \mathrm{l}$ de OPD y $\mathrm{H}_{2} \mathrm{O}_{2}$ en buffer urea $\mathrm{pH}$ 5.0. Se frenó la reacción a los 15 minutos, con $50 \mu \mathrm{l}$ de HCL $2.5 \mathrm{~N}$. La lectura de los valores de absorbancia se realizó a 492nm en un lector de ELISA Multi-Skan Plus MK-II.

El valor del punto de corte se calculó (VC) con el promedio aritmético de la sumatoria de los valores de absorbancia de los sueros control negativos ( $\mathrm{X} C N)$ más dos desviaciones estándar (2DS). Punto de Corte: 0,289. Las muestras con absorbancias mayores al valor del punto corte se consideraron positivas. Las 102 muestras de sangre total, se evaluaron bajo las condiciones establecidas previamente para el ELISA.

\section{Examen Coproparasitológico}

Las 159 muestras coprológicas se procesaron con la técnica de concentración de formol-éter, y el examen coproparasitológico se realizó con el objetivo de 40X por triplicado y doble ciego, para la identificación de huevos de Taenia $\operatorname{spp}(42)$.

\section{Análisis estadístico}

Se tabuló la información obtenida en la encuesta estructurada con las condiciones sociodemográficas, los resultados de la técnica de diagnóstico serológico en porcinos y la coprología para teniasis humana, para el análisis estadístico se empleó el programa estadístico SPSS V ${ }^{22}$ realizando un análisis univariado y estableciendo la relación estadística con un estudio bivariado utilizando la prueba de chi cuadrado.

\section{Resultados}

Los resultados revelaron una seroprevalencia de cisticercosis porcina del 17\% (17/102), se observó que las hembras correspondieron al mayor número de casos positivos para la prueba, con un total de 11/102 para un 10,8\% apreciándose que la variable presentó asociación estadística de seropositividad $p \leq 0.005$. En cuanto a la distribución por raza, 11/102 se identificaron con títulos de anticuerpos en raza criolla, $10,8 \%$ con un valor de $p \leq 0.005$. En cuanto al grupo etario, el mayor número de seropositivos fue de dos a nueve meses con un total de 13/102 equivalente a $12,7 \%$. Otra de las variables evaluadas fue el peso, el grupo con mayor número de inmunoreactivos para la entidad fue el de 11-24 kilos con 7,8\% (8/102). 
Tabla 1. Valores porcentuales de seroprevalencia y posible asociación con las variables incluidas en el estudio, que presentaron significancia estadística en la población porcina del área urbana del municipio de Coyaima (Tolima)

\begin{tabular}{|c|c|c|c|c|c|c|}
\hline Variable & Número de muestras & $\%$ & Seropositivos & $\%$ & Valor de $\mathrm{P}$ & IC95\% \\
\hline Etapa de crecimiento & & & & & $\leq 0.05$ & \\
\hline Lactancia & 21 & 20,6 & 7 & 6,8 & & \\
\hline Levante & 62 & 60,8 & 10 & 9,8 & & \\
\hline Ceba & 19 & 18,6 & 0 & 0 & & \\
\hline Lugar de crianza & & & & & $\leq 0.05$ & \\
\hline Dentro de la casa & 72 & 70,6 & 13 & 12,7 & & \\
\hline En el campo & 30 & 29,4 & 4 & 3,9 & & \\
\hline Sistema de Crianza & & & & & $\leq 0.05$ & \\
\hline Campo abierto & 34 & 33,3 & 5 & 4,9 & & \\
\hline Cochera & 68 & 66,7 & 12 & 11,8 & & \\
\hline Quién cría al cerdo & & & & & $\leq 0.05$ & \\
\hline Amigo & 3 & 2,92 & 1 & 1,0 & & \\
\hline Esposa & 48 & 47 & 13 & 12,7 & & \\
\hline Familia & 36 & 35,3 & 1 & 1,0 & & \\
\hline Padre de familia & 15 & 14,7 & 2 & 2,0 & & \\
\hline Alimentación & & & & & $\leq 0.05$ & \\
\hline Desperdicios & 60 & 58,8 & 14 & 13,7 & & \\
\hline Desperdicios y campo abierto & 6 & 5,8 & 0 & 0,0 & & \\
\hline Campo abierto & 8 & 7,8 & 0 & 0,0 & & \\
\hline Concentrado y desperdicios & 28 & 27,5 & 3 & 2,9 & & \\
\hline Fuentes de Agua & & & & & $\leq 0.05$ & \\
\hline Con tratamiento & 24 & 23,5 & 4 & 3,9 & & \\
\hline sin tratamiento & 78 & 76,5 & 13 & 12,7 & & \\
\hline \multicolumn{7}{|l|}{ Presencia de \#s animales } \\
\hline No & 9 & 8,8 & 2 & 2,0 & & \\
\hline $\mathrm{Si}$ & 93 & 91,2 & 15 & 14,7 & & \\
\hline \multicolumn{7}{|l|}{ Uso de desparasitante } \\
\hline No & 15 & 14,7 & 3 & 2,9 & & \\
\hline $\mathrm{Si}$ & 87 & 85,3 & 14 & 13,7 & & \\
\hline Periodicidad & & & & & $\leq 0.05$ & \\
\hline 1-4 veces al año & 89 & 87,3 & 15 & 14,7 & & \\
\hline Nunca & 13 & 12,7 & 2 & 2,0 & & \\
\hline Vacuna peste porcina clásica & & & & & $\leq 0.05$ & \\
\hline No & 57 & 56 & 12 & 11,8 & & \\
\hline $\mathrm{Si}$ & 45 & 44,1 & 5 & 4,9 & & \\
\hline Consulta veterinaria & & & & & $\leq 0.05$ & \\
\hline No & 36 & 35,3 & 6 & 5,9 & & \\
\hline $\mathrm{Si}$ & 66 & 64,7 & 11 & 10,8 & & \\
\hline Sacrificio del cerdo & & & & & $\leq 0.05$ & \\
\hline Casa & 98 & 96,1 & 16 & 15,7 & & \\
\hline Matadero & 4 & 3,92 & 1 & 1,0 & & \\
\hline Destino final del cerdo & & & & & $\leq 0.05$ & \\
\hline Autoconsumo & 4 & 3,92 & 0 & 0,0 & & \\
\hline Autoconsumo y venta & 37 & 36,3 & 7 & 6,9 & & \\
\hline Venta & 44 & 43,1 & 8 & 7,8 & & \\
\hline Comercialización en pie & 17 & 16,6 & 2 & 2,0 & & \\
\hline \multicolumn{7}{|l|}{ Comercialización de la carne } \\
\hline En la misma vereda & 92 & 90,2 & 17 & 16,7 & & \\
\hline Otras veredas & 10 & 9,8 & 0 & 0,0 & & \\
\hline
\end{tabular}




\section{Variables del Estudio}

Fuentes de agua

Los afluentes que suministran agua a este municipio son los Ríos Saldaña y Magdalena, pero las comunidades no cuentan con un sistema de acueducto y alcantarillado adecuado, esta variable fue estadísticamente significativa respecto a los resultados hallados de seropositivos para la presencia de anticuerpos anticisticercos con un $p \leq 0,05$. El 23,3\% (24/102) de los tenedores argumentaron poseer agua con algún tipo de tratamiento para el consumo de sus animales, en tanto que el 76,5\% (78/102) manifestaron no disponer de este. De este último grupo se identificaron $12,7 \%(13 / 102)$ con anticuerpos anticisticerco. En tanto que los que contaban con suministro de agua tratada evidenciaron positivos (4/102).

Tipo de crianza, sistema de crianza y alimentación

\section{Etapa de desarrollo}

Se halló significancia estadística para la fase de lactancia y levante con valor de $\mathrm{p} \leq 0,05$, de los cuales, para la primera, se presentó $6,8 \%$ de seropositivos y $9,8 \%$ para la segunda; en tanto que en la ceba ninguno de los animales evaluados presentó títulos de anticuerpos contra la fase larval de T. solium.

\section{Sistema y lugar de crianza}

Estas variables presentaron significancia estadística con valor de $\mathrm{p} \leq 0,05$ y se halló que el 66,6\% (68/102) se llevó a cabo en sistema de cochera y el 33,3\% (34/102) en campo abierto. En cuanto a la presencia de anticuerpos el 11,7\% (12/102) fueron para animales confinados en porquerizas y 5\% (5/102) para la siguiente condición. El análisis en lo referente en cuanto a la localización de las porquerizas, si es en el lugar de residencia de los propietarios, se identificó que el $70,6 \%(72 / 102)$ es en el domicilio donde el $12,7 \%$ $(13 / 102)$ de los animales evaluados presentaron títulos de anticuerpos y 29,4\% (30/102) se lleva a cabo en la zona rural (4/102), con un $3,9 \%$ de seropositividad.

\section{Tipo de alimentación}

El 58,8\% (60/102) se realiza con desechos domésticos de los cuales el 13,7\% (14/102) fueron positivos. Para el caso de suministro de dietas mixtas (desechos domésticos y concentrado), $27,5 \%$ (28/102) donde se encontraron tres casos positivos correspondientes al 2,9\%.

\section{Desparasitación}

El uso de antiparasitarios se evidenció en un $85,3 \%$ (87/102) en los ejemplares, pero llama la atención que el 13,7\% (14/102) fueron seropositivos y en el $14,7 \%$ (15/102), sus propietarios manifestaron no cumplir con este esquema, entre estos se halló un $2,9 \%$ con anticuerpos, aunque se les suministran los antiparasitarios con una periodicidad de una a cuatro veces al año en el 87,3\% (89/102), de los cuales $14,7 \%$ fueron diagnosticados seropositivos; al igual que en el grupo de los que no recibieron tratamiento, $12,7 \%$ (13/102) del cual en el 2,0\% se evidenció reacción positiva.

\section{Vacuna viral para moquillo}

Se identificó que el 44,1\% (45/102) cumplieron con la condición de vacunación contra la peste porcina clásica, el grupo de los no vacunados fue el 56\% (57/102) hallándose el $17 \%$ de seropositivos para ambos grupos.

\section{Consulta médica veterinaria}

El 64,7\% (66/102) de los tenedores manifestaron contar con la asistencia; sin embargo, se observó que el $10,8 \%(11 / 102)$ presentaron anticuerpos contra el estadio larval; el 35,3\% (36/102) expresaron no realizar consulta médica veterinaria, de los cuales el 5,9\% fue seropositivo.

\section{Lugar de sacrificio y destino de la carne}

Finalmente, entre las variables evaluadas, para el sacrificio de los cerdos se tomaron en cuenta dos aspectos, el realizado en el lugar de vivienda del propietario y/o en el centro de sacrificio. Siendo el lugar de residencia $96,1 \%$ con un total de 16 seropositivos $(15,7 \%)$, y un $1 \%$ para los que acuden al centro de beneficio municipal. Con respecto al destino final de la carne, se encontró que la venta era del $43,1 \%$, el autoconsumo y venta de $36,3 \%$, el autoconsumo exclusivamente del $3,92 \%$ y por último la comercialización en pie de $16,6 \%$. Otra variable que se tomó en cuenta, es el lugar de comercialización de la misma, manifestando el 90,2\% venderla domiciliariamente. Los valores porcentuales de seroprevalencia y las variables incluidas en el estudio con significancia estadística se presentan en la tabla 1. 
Otro aspecto evaluado fue la actividad de manutención; siendo el ama de casa la de mayor contacto con los animales $47 \%$ (48/102), en su respectivo orden el padre de familia $14,7 \%(15 / 102) ; 35,3 \% \quad(36 / 102)$ otros miembros de la familia y el 2,92 (3/102) un tercero. Las características descriptivas y valores porcentuales de seroprevalencia de la población porcina evaluada se relacionan en la tabla 2.

Tabla 2. Características descriptivas y valores porcentuales de seroprevalencia de la población porcina del área urbana del municipio de Coyaima (Tolima)

\begin{tabular}{|l|c|c|c|c|c|}
\hline Variable & Número de muestras & $\%$ & Seropositivos & $\%$ & Valor de P IC 95\% \\
\hline Raza & & & & & \\
\hline Criollo & 54 & 53 & 11 & 10,8 & \\
\hline De Raza & 48 & 47 & 6 & 5,8 & \\
\hline Edad & & & & & \\
\hline $2-9$ meses & 72 & 70,5 & 13 & 12,7 & \\
\hline 10-14 meses & 27 & 26,5 & 4 & 3,9 & \\
\hline > a 15 meses & 3 & 2,92 & 0 & 0 & \\
\hline Peso & & & & & \\
\hline $1-10$ kilos & 32 & 31,4 & 5 & 4,9 & \\
\hline $11-24$ kilos & 41 & 40,2 & 8 & 7,8 & \\
\hline$>$ a 25 kilos & 29 & 28,4 & 4 & 3,9 & \\
\hline Género & & & & & \\
\hline Hembra & 56 & 55 & 11 & 10,8 & \\
\hline Macho & 46 & 45,1 & 6 & 5,8 & \\
\hline
\end{tabular}

Parasitismo intestinal en personas criadoras de cerdos del área urbana del Municipio de Coyaima (Tolima). A las 159 personas participantes en el estudio se les realizó un examen coproparasitológico, de las cuales el $43.3 \%$ (69/159) fueron positivas por presentar al menos una entidad parasitaria. Los agentes etiológicos y los valores porcentuales se presentan en la tabla 3.

\section{Discusión}

En el presente estudio se halló posible asociación de diferentes variables con la cisticercosis porcina en el municipio de Coyaima. De acuerdo al estudio realizado, se determinó una seroprevalencia del $17 \%$. Estudios en los años 90 por el Instituto Nacional de Salud evidenciaron una prevalencia para cisticercosis porcina de $25.64 \%$ y superando la de cisticercosis humana del $12.4 \%(22)$, lo que permite afirmar que es un riesgo para la salud pública, ya que se involucran aspectos socio-económicos y culturales en el ciclo de vida del parásito, siendo las actividades humanas las directamente implicadas en la reproducción y dispersión del parásito.
Para el desarrollo del metacéstodo de T. solium, en el ciclo natural son indispensables los cerdos como hospederos intermediarios y cursar asintomáticos la infección, aunque algunos autores manifiestan que pueden presentar sintomas como: anorexia, diarrea y fiebre entre otros $(8,22,43-45)$.

La colecta de la muestra de sangre por impregnación en discos de papel de filtro, presenta ventajas, ya que no genera estrés en los animales, presenta comodidad para el transporte y la conservación, no es necesario el desplazamiento de equipos y elementos costosos $(29,30)$. La prueba de ELISA estandarizada y el empleo de la fracción proteica de $53 \mathrm{kDa}$ como antígeno, le confiere valores de sensibilidad del $100 \%$, especificidad de $99.1 \%$ y valores predictivos positivo y negativo del $98.5 \%$ y $100 \%$ (41).

Los aspectos más significativos hallados en la propagación del complejo teniasis/cisticercosis en la comunidad de Coyaima fueron: el no contar con buenos sistemas para disposición de excretas, factor que está asociado con la teniasis en todas las zonas endémicas del mundo debido a que el humano es el único responsable de la 
Tabla 3. Valores porcentuales de prevalencia de parasitismo intestinal en personas criadoras de cerdos para el periodo 2012 y 2013 del área urbana del municipio de Coyaima Tolima

\begin{tabular}{|c|c|c|c|}
\hline Entidad Parasitaria & N. de casos & $\%$ Prevalencia & IC95\% \\
\hline \multicolumn{4}{|l|}{ Helmintos } \\
\hline Uncinaria & 8 & 5,0 & \\
\hline Taenia spp & 3 & 1,9 & \\
\hline Ascaris lumbricoides & 3 & 1,9 & \\
\hline Dipylidium caninum & 1 & 0,6 & \\
\hline \multicolumn{4}{|l|}{ Protozoos } \\
\hline Entamoeba coli & 28 & 17,6 & \\
\hline Complejo E. histolytica dispar/moshkouskii & 49 & 30,8 & \\
\hline Endolimax nana & 43 & 27,0 & \\
\hline Iodamoeba butschlii & 22 & 13,8 & \\
\hline Cyclospora cayetanensis & 44 & 27,7 & \\
\hline Neobalantidium coli & 20 & 12,6 & \\
\hline Cystoisospora belli & 15 & 9,4 & \\
\hline Giardia spp & 7 & 4,4 & \\
\hline Chilomastix mesnili & 5 & 3,1 & \\
\hline Entamoeba hartmanni & 5 & 3,1 & \\
\hline Blastocystis spp & 1 & 0,6 & \\
\hline
\end{tabular}

dispersión de los huevos del parásito y el no poseer planta para tratamiento de agua, acueducto y alcantarillado $(11,46,47)$ ya que los huevos tienen capacidad de sobrevivir en aguas estancadas o con corriente natural que fluya con continuidad (48), lo que hace intuir que esta se la posible vía de contaminación para los cerdos, basado en lo manifestado por el $76.5 \%$ de la comunidad de no tener acceso un recurso hidrico potable.

Otro aspecto a tener en cuenta, es la crianza de cerdos, en condiciones que le permiten acceso a las heces humanas acompañados de hábitos inadecuados en su tenencia como alimentación con desperdicios, variable que en el presente estudio evidenció que más del $50 \%$ de la población porcina es alimentada con sobras exclusivamente y de éstos el 13,7\% fueron animales seropositivos, y algunos se liberaban a campo abierto para la búsqueda de alimento y agua (20).

Con respecto a la responsabilidad de crianza se identificó al género femenino como el de mayor porcentaje (47\%), variable que en diversos estudios lo evidencian como un factor de riesgo inminente para adquirir la infección debido a que las mujeres se encargan de la manutención de los animales o son amas de casa, preparan los alimentos, entre otras actividades, por lo que el riesgo de contaminarse es mayor en relación al género masculino $(49,50)$.

Otro aspecto a tener en cuenta es el bajo control sanitario de la carne en el faenamiento y sacrificio de los animales, la venta y malos hábitos de consumo siendo estos la baja cocción e incluso el consumo de carne cruda, prácticas que también favorecen la infección por esta entidad parasitaria (51).

La presencia de teniásicos en la comunidad evaluada en $1,9 \%$, (3/102) constituye un factor de riesgo para la adquisición de la cisticercosis tanto porcina como humana y con posible desarrollo de Neurocisticercosis $(52,53)$, ya que una persona teniásica libera los huevos del parásito y aporta la forma infectante, además que una sola proglótide grávida puede liberar al menos 50.000 de éstos, los cuales pueden ser viables en condiciones normales del medio ambiente para generar los estadios infectivos que dan origen a la fase larval sin lo cual no sería posible la continuidad del ciclo.

En el estudio se implementó un examen coproparasitológico con el fin de identificar huevos de Taenia spp, los 
cuales no permiten discriminación entre una y otra especie puesto que la estructura es similar, solo es posible diferenciar en el caso que se disponga del estadio adulto de la estructura de fijación o escólex, que para el caso de $T$. solium, posee doble corona de ganchos con la cual se adhiere al intestino delgado humano o la evacuación de proglótides grávidos expulsadas en las heces y que bajo observación al microscopio estereoscopio con poco aumento se aprecian las ramas uterinas que en número de 8-12 corresponden a $T$. solium, y superior a este (12-24) a $T$. saginata (54).

Sin embargo, por las diferentes variables estimadas con vínculo ecoepidemiológico de esta parasitosis y la presencia de anticuerpos anticisticerco en las muestras evaluadas que corrobora la existencia de cisticercosis porcina en el municipio, permite argumentar la presencia de T. solium, en esta población, así como se demuestra con la presencia de tres personas con huevos para el género.

Con lo reportado por otros autores y lo hallado en esta investigación, se corrobora que Colombia sigue siendo parte del grupo de países endémicos para el parásito y coexiste en zonas con condiciones favorables y expuestas a los factores de riesgo asociados al agente etiológico para que se dé la presencia del binomio teniasis/cisticercosis $(20,46)$.

Otro hallazgo relevante fue la presencia de Neobalantidium coli(55), en la población humana con $12,6 \%(20 / 159)$, único protozoario ciliado que parasita al ser humano, siendo un reporte mayor al de otros autores que lo estiman entre $0,02-1 \%$ en el mundo, aunque la encuesta nacional de parasitismo para Colombia 2012-2014 sólo hallo un caso en población infantil en la provincia Norandina (56), se han reportado brotes de neobalantidiasis en lugares donde no hay ningún contacto con porcinos pero si hacinamiento y bajas condiciones higiénico sanitarias como prisiones, orfanatos $(57,58)$, en Estados Unidos y Canadá esta parasitosis es poco frecuente y siempre han correspondido a casos importados por personas que han referido realizar tránsito por áreas endémicas $(59,60)$ aunque se reportan valores particulares del $28 \%$ de prevalencia en criadores de cerdos en Nueva Guinea y del $67 \%$ en cerdos criados domésticamente y en granjas, en seis comunidades de dos estados de Venezuela (61). Los brotes de infecciones puede tener una justificación en la relación entre los procesos de crianza y el tenedor, ya en varios estudios se argumenta una posible contaminación directa a través de agua, alimento o deficiencia en condiciones higiénico sa- nitarias, infección parasitaria que es asintomática en los cerdos pero puede ser sintomática en el ser humano con cuadro disentérico acompañada de constipación, dolor abdominal secundario a la invasión del intestino grueso por trofozoitos y en ocasiones fulminante, con lesiones en el intestino, evidenciadas por la presencia de moco y sangre en las heces (62).

\section{Conclusiones}

Con el empleo de la técnica de toma de sangre seca total en PF y la optimización de una prueba de ELISA se pudo determinar la prevalencia de anticuerpos anticisticercos en porcinos del $17 \%$, demostrando el carácter endémico de esta entidad parasitaria zoonótica en la zona urbana del municipio de Coyaima (Tolima), por lo que se hace necesaria la formulación de medidas de control y prevención. Se observó mayor asociación con fuentes de agua, personas teniásicas, condiciones ambientales, tipo de alimentación.

La prevalencia general de parasitismo intestinal en los criadores fue de $43.3 \%$, y se encontraron tres personas de 159con huevos de Taenia spp y las fuentes de agua $y$ tenencia de porcinos como variables estadísticamente significativas $(p \leq 0,005)$

El complejo Teniosis/cisticercosis es un serio problema de salud pública en la comunidad coyaimuna y sería muy importante que comenzaran iniciativas para la vigilancia y control de esta parasitosis, así como lo plantea la reunión de expertos realizada por la OMS (6).

\section{Agradecimientos}

Al Grupo de Investigación en Parasitología y Microbiología Tropical- GIMPAT, de la Universidad INCCA de Colombia. Al laboratorio de Investigación en Parasitología de la Universidad INCCA de Colombia, Programa Profesional de Biología. A la comunidad del Municipio de Coyaima por su participación en el proyecto.

Al Centro de Estudios en Microbiología y ParasitologíaCEMPA de la Facultad de ciencias de la salud programa de Medicina de la Universidad del Cauca.

\section{Declaración de conflicto de Intereses}

Los investigadores manifestamos que esta investigación no presenta ningún conflicto de interés, que todos los participantes en el estudio fueron debidamente notifica- 
dos, que las muestras biológicas y la información que este contiene se manejaron con estricta reserva y tratamiento bioético

\section{Fuente de financiación}

Este estudio fue financiado por la Universidad INCCA de Colombia aprobado en la Convocatoria interna 007/022010/2011 y desarrollado por el Grupo de investigación de Parasitología y Microbiología tropical, GIPAMT de la Universidad INCCA de Colombia y El Centro de Investigaciones en Microbiología y Parasitología - CEMPA de la Facultad de Medicina de la Universidad del Cauca.

\section{Referencias}

1. Welburn I, Beange M, Ducrotoy J and Okello AL. The neglected zoonoses the case for integrated control and advocacy. Clin Microbiol Infect.2015;21:433-443. Disponible en: http://dx.doi.org/10.1016/j.cmi.2015.04.011

2. Hotez PJ, Bottazzi ME, Franco-Paredes C, Ault SK and Periago MR. The Neglected Tropical Diseases of Latin America and the Caribbean: A Review of Disease Burden and Distribution and a Roadmap for Control and Elimination. PLoS Negl Trop Dis.2008;2(9):e300. Disponible en: http://dx.doi.org/10.1371/journal.pntd.0000300

3. Del Bruto $\mathrm{OH}$, García HH. Taenia solium Cysticercosis-The lessons of history. J Neurological Sciences.2015;359:392-395. Disponible en: http://dx.doi. org/10.1016/j.jns.2015.08.011

4. García HH, González AE, Evans Caw, Gilman RH. Taenia solium cisticercosis. Lancet. 2003;326:547-56. Disponible en: http://dx.doi.org/10.1016/S01406736(03)14117-7

5. Flisser A, Sarti E, Lightowlers M and Schahtz P. Neurocysticercosis: regional status epidemiology, impact and control measures in the Americans. Acta Tropical.2003;(87):43-51. Disponible en: http://dx.doi. org/10.1016/S0001-706X(03)00054-8

6. Román G, Sotelo, Del Brutto O, Flisser A, Dumas M, Wadia N, Botero D, Cruz M, Garcia H, De Bittencourt PR, Trelles L, Arriagada C, Lorenzana P, Nash TE and Spina-França, A. A Proposal to declare neurocysticercosis an international reportable disease. WHO IRIS.2000;78(3):(399-406). PMID:25123870.Disponible en: http://www.ncbi.nlm. nih.gov/ pubmed/ ? term $=$ Roman $+2000+$ Neurocystic ercosis

7. Sierra O, Cardozo E. Seroprevalencia de cisticercosis porcina en el municipio de Galeras, departamento de Sucre, mediante la prueba de inmuno ensayo Elisa con la fracción polipeptídica de $53 \mathrm{kDa}$ del metacestodo Taenia soli-
um.2007;Disponible en: http://repositorio.unisucre.edu. co/bitstream/001/49/2/T616.964079\%20C268.pdf

8. Román G, Sotelo J, Del Brutto O, Flisser A, Dumas M, Wadia N, Botero D, Cruz M, Garcia H, De Bittencourt PR, Trelles L, Arriagada C, Lorenzana P. Nash TE, Spina-França, A. A Proposal to declare neurocysticercosis an international reportable disease. WHO IRIS.2000;78(3):(399-406).PMID:25123870. Disponible en: http://www.ncbi.nlm.nih.gov/ pubmed/ ? term= Roman $+2000+$ Neurocysticercosis

9. Sakai SH. Breve Informe: Seroprevalencia de Cisticercosis Porcina Taenia solium en el estado de Bahía al norte de Brasil. Am. J. Trop Med.2001;64(5,6):268-269.

10. Maravilla P, Souza V, Romero C and Flisser A. Isozyme analysis of Taenia solium isolates from Mexico and Colombia.2003;(9):30-40.(Ed. Dec) Men Inst Oswaldo Cruz. Epub. Disponible en: http://www.ncbi.nlm.nih.gov/pub$\mathrm{med} /$ ?term $=$ maravilla $+2003+$ cysticercosis

11. Garcia H, Gonzalez A, Gilman R. Diagnosis, treatment and control of Taenia solium cysticercosis. Lancet.2003;362,547-556. Disponible en: http://www. ncbi.nlm.nih.gov/pubmed/14501993

12. OMS. Teniasis/Cisticercosis Nota descriptiva $N^{\circ} 376$. Recuperado el 17 de Noviembre de 2015. Organización Mundial de la Salud: Disponible en: http://www.who.int/ mediacentre/factsheets/fs376/es

13. Agudelo P, Restrepo B y Palacio L. Conocimiento y Prácticas sobre Teniasis - Cisticercosis en una Comunidad Colombiana. Rev. Salud pública.2009;11(2):191-199. Disponible en: http://www.scielosp.org/pdf/rsap/v11n2/ v11n2a04.pdf

14. OPS/OMS. Organización Panamericana de la Salud. Epidemiología y control de la taeniosis y cisticercosis en América Latina. Washington, D.C.1994;Versión1.0. Disponible en: http://www.ejournal.unam.mx/rfm/no45-2/ RFM45204.pdf

15. Engels D, Urbani C, Belotto A, Meslin F, Savioli L. The control of human (neuro) cysticercosis: which way forward. Acta Trop.2003;87,177-182. Disponible en: http:// helminto.inta.gob.ar/A\%20Cisticercosis/The\%20control\%20of\%20human\%20(neuro)cysticercosis.pdf

16. Nitin S, Nuzhat H, Vimala V, Jamal M, Mazhar H. Seroprevalence of cysticercosis in North Indian population. Asian Pacific Journal of Tropical Medicine.2010;3(8):589-593. Doi:10.1016/S1995-7645(10)60144-7. Disponible en: http://www.sciencedirect.com.ezproxy.unal.edu.co/science/article/pii/S1995764510601447

17. Sarti E, Román G, Sotelo J, Del Brutto O, Flisser A, Dumas M, Wadia N, Botero D, Cruz M, Garcia H, de Bittencourt PR, Trelles L, Arriagada C, Lorenzana P, Nash TE, Spina-França A. Development and evaluation of a health edu- 
cation intervention against Taenia solium in a rural community in Mexico. Am. J:Trop. Med.Hyg.1997;6,127-132. http://www.ncbi.nlm.nih.gov/ pubmed/ ?term=Development++ evaluation+of+a+health+education+intervention+against+Taenia +solium +in +a+rural+community+in+Mexico.

18. Zbigniew P, Allan J, Sarti E. Control of Taenia solium taeniasis/cysticercosis: From research towards implementation, International Journal for Parasitology.2005;(35):11-12. Disponible en: http://www.sciencedirect.com.ezproxy. unal.edu.co/science/article/pii/S0020751905002754

19. González A, García H, Rodríguez S, González G, Llanos Z, Tsang R, Gilman H. Epidemiology and control of cysticercosis in Peru. Acta Trop.2010;(83):103-109. doi:http:// dx.doi.org/10.1590/S172646342010000400016.

20. Flórez A, Pastrán S, Vargas N, Beltran M, Enriquezy Muñoz L. Cisticercosis en Colombia Estudio de seroprevalencia 2008-2010. Acta Neural Colomb.2013;29(2):73-86.Disponible en: http://www.scielo.org.co / scielo,php ? scrip $\mathrm{t}=$ sci_arttext\&pid $=$ S0120-87482013000200003.

21. Vásquez L, Giraldo J, Agúdelo P, Campo V, Vergara D. Experiencia para el control de la cisticercosis en el departamento del Cauca. Biomédica.2011;31(sup.3):3-315. Disponible en: http://www.revistabiomedica.org/index. php/biomedica/article/viewFile/536/665

22. Serrano J, Prada F, Nicholls R, Duque S y López M. Determinacion De La Prevalencia de cisticercosis porcina en cuatro veredas del municipio de Coyaima. Biomedica.1993;13:129-135.Disponible en: http://www.revistabiomedica.org/ index.php/ biomedica/ article/ viewFile/ 2058/2094

23. Agudelo P, Palacio LG. Prevalencia de anticuerpos para Taenia solium en humanos y cerdos en una zona endémica Colombiana. Rev Neurol.2003;36:706-709. Disponible en: http://www.ncbi.nlm.nih.gov/pubmed/?term=agudelo+2003+Taenia+solium.

24. Dorny P, Brandt J, Zoli A, Geerts H. Immunodiagnostic tools for human and porcine cysticercosis. Acta Tropica.2003;87(1):79-86. Disponible en: http://www.sciencedirect.com.ezproxy.unal. edu.co/ science/ article/ pii/ S0001706X03000585

25. Romani ECG. Manual de Procedimientos para diagnóstico serológico de las Zoonosis parasitarias. (S. Edición, Ed.) Serie de normas técnicas.2010;32:59-62. Disponible en: http://www.ncbi.nlm.nih.gov/pubmed/19912602

26. Tsang V, Brand J, Boyer A. An enzyme-linked immunoelectrotransfer blot assay and glycoprotein antigens for diagnosing human cysticercosis (Taenia solium). J. Infect.1989;50-59. Disponible en: http://www.ncbi.nlm. nih.gov/pubmed/2909643

27. Wilson M, Bryan R, Fried J, Ware D, Schantz P, Pilcher J and Tsang, V. Clinical evaluation of the cysticercosis enzyme-linked immunoelectrotransfer blot in patients with neurocysticercosis. J. Infect.1991;(164):1007-1009. Disponible en: http://www.ncbi.nlm.nih.gov/pubmed /1940452

28. Ramahefarisoa R, Rakotondrazaka M, Jambou R, Carod J. Comparison of ELISA and PCR assays for the diagnosis of porcine cysticercosis. Veterinary Parasitology.2010;173(3-4):336-339. Disponible en: https:// www.ncbi.nlm.nih.gov/pubmed/20542639

29. Mei J, Alexander J, Adam B, Hannon W, Souza V, Nitin S. Use of filter paper for the collection and analysis of human whole blood specimens. J Nutr.2001;131(5):3342. Disponible en: http://www.ncbi.nlm.nih.gov/ pubmed/11340130

30. Andriamandimby S, Heraud J, Randrianasolo L, Rafisandratantsoa T, Andriamamonjy S, Vincent R. Driedblood spots: a cost-effective field method for the detection of chikungunya virus circulation in remote areas. PLoS Negl. Trop.2013;(7):p.e 2339 Disponible en: http:// journals.plos.org/plosntds/article?id=10.1371/journal. pntd.0002339

31. Wasniewski M, Barrat J, Combes B, Guiot A, Cliquet C. Use of filter paper blood samples for rabies antibody detection in foxes and raccoon dogs. J Virol Methods.2014;13:11-6. doi: 10.1016/j.jviromet.2014.04.005.

32. Restrepo I, Mazo L, Salazar M, Montoya M, Botero J. Evaluación de tres técnicas coproparasitoscópicas para el diagnóstico de geohelmintos intestinales. Revista Iatreia. 2013;26(1):15-24. Disponible en: http://www.scielo.org. $\mathrm{co} / \mathrm{pdf} / \mathrm{iat} / \mathrm{v} 26 \mathrm{n} 1 / \mathrm{v} 26 \mathrm{n} 1 \mathrm{a02}$.pdf

33. Duque S, Guerrero R, Nicholls R, López M. Examen coproparasitológico en niños: comparación de resultados obtenidos por dos métodos en dos instituciones de Santafé de Bogotá, D.C. Revista Biomédica.1994;14(1):39-47. Disponible en: http://www.revistabiomedica.org/index. php/biomedica/article/view/2084/2118

34. Zierdt W. A simple device for concentration of parasite eggs, larvae, and protozoa. Am j Clin Pathol.1978;70:89-93. Disponible en: https://www.ncbi.nlm.nih.gov/pubmed/ 567940

35. McMillan A, Mcneillage G. Comparison of the sensivity of microscopy and culture in the laboratory diagnosis of intestinal protozoal infection. J Clin Pathol.1984;37:809-811. Disponible en: http://www.ncbi.nlm.nih.gov/pubmed/?term =Comparison +of +the+sensivity +of + microscopy+and +culture+in+the+laboratory+diagnosis+of+intestinal+protozoal+infecton

36. Truant A, Elliot S, Kelly M, Smith J. Comparison offormalinethylether sedimentation, formalin-ethylaetate sedimentation, and zinc sulfate flotation techniques 
for detection of intestinals parasites. $\mathrm{J}$ Clin Microbiol.1981;13:882-884. Disponible en: http://www.ncbi. nlm.nih.gov/pubmed/?term=Comparison+offormalinethylether+sedimentation\% $2 \mathrm{C}+$ formalinethylaetate + sedimentation\% $2 \mathrm{C}+$ and + zinc+sulfate+flotation+techniques+for+detection+of+intestinals+parasites

37. González C, Giraldo J. Prevalencia de parasitos intestinales en caninos (Canis lupus familiaris) en el municipio de Coyaima (Tolima). Revista Med.2015;23(2):24-34. DOI: http://dx.doi.org/10.18359/rmed.1743

38. Moncaleano J. Plan de desarrollo municipal todos por Coyaima (2012-2015).2012. Disponible en: http://www. coyaima-tolima.gov.co/apc-aa-files/ 326666316366663 56631353165613764/ pd-2012-2015-coyaima-domingo-29-v7-1111.pdf

39. PBOT. Demografía. Plan Básico de Ordenamiento Territorial - Coyaima. Alcaldía Municipal de Coyaima Tolima.2010. Disponible en: http://www.coyaima-tolima. gov.co/ apc-aa-files/ 32666631636666356631353 165613764/DIAGN_STICO_P.B.O.T.pdf

40. Registro Mercantil. Alcaldías y Gremios de la Jurisdicción. Secretaria de Planeación Infraestructura y Desarrollo alcaldía de Coyaima.2013. Disponible en: http://www. ccsurortolima.org.co/portal/images/Informe_Econ_ mico_2013_.pdf

41. Giraldo J. Valoración inmunológica con sueros de porcinos de los polipeptidos de 64,53 y 32KDa del metacéstodo de Taenia solium. Universidad de los Andes.2000. Disponible en: http://classify.oclc.org/classify2/ClassifyDemo?owi $=2588663333$

42. Estrada E. Manual de Procedimientos de laboratorios para el diagnóstico de los parásitos intestinales del hombre. (I. N. Salud, Ed.) Serie de Normas Técnicas.2003;37:10-16. Disponible en: http://www.ncbi. nlm.nih.gov/pubmed/14765651

43. Pawlowski Z, Schultz M. Teniasis and cysticercosis (Taenia saginata). Advances in Parasitology.1972;10:269-343. Disponible en: http://www.ncbi.nlm.nih.gov/pubmed/ 4559145

44. OMS/OPS. Epidemiologia y control de la teniasis y cisticercosis en América Latina. WHO. 1993;Ver2.0, PNSP/ 9128 Disponible en: http://www.veterinaria.org/revistas/redvet/n111107/110701.pdf

45. Walteros D. Cisticercosis: situación de parasitosis. Informe Final de Evento Cisticercosis.2009;Ver1.013.09. Disponible: http://www.ins.gov.co/ lineas- de- accion/ Subdireccion-Vigilancia/Informe\% 20 de \% 20 Evento \% 20 Epidemiolgico/ Cisticercosis \% 202009.pdf

46. Flórez A, Pastrán M, Peña A, Peña P, Benavides A, Villarreal A, Rincón A, Garzón I, Muñoz L y Guasmayan L. Cisticercosis en Boyacá, Colombia: estudio de seroprev- alencia. Acta Neural Colomb.2011;27:9-18. Disponible en: http://www.ncbi.nlm.nih.gov/ pubmed/? term=control+ of+human+ (neuro)+ cysticercosis\%3A+ which+ way+ forward

47. Komba E. Prevalence of porcine cysticercosis and associated risk factors in smallholder pig production systems in Mbeyra region, southern highlands of Tanzania. Vet Parasitol. 2013;6(198):3-4. doi: 10.1016/j.vetpar.2013.09.020. Disponible en: http://www.ncbi.nlm. nih.gov/pubmed/?term=Komba+2013+cysticercosis

48. Jubb KP. Patología de los animales domésticos. Academic Press Inc. 1992;1:255-256.

49. Franco C, Giraldo J, Vásquez R. Detección de anticuerpos anticisticerco en pacientes que asistieron a consulta médica durante el periodo 2009 a la Liga Contra la Epilepsia. Capitulo Cauca, Revista UNINCCA.2012;18(1):79-93. Disponible en: https://www.unincca.edu.co/images/stories/pfd/revista-cientifica-v18-1. pdf

50. Ayala E, Medina S, Morales J. Cisticercosis humana y sus asociación con factores epidemiológicos y clínicos en los Andes centro y sur de Perú. Revista epidemiológica del Perú.2014;18(1):1-6. Disponible en: http://www.ncbi. nlm.nih.gov/pubmed/?term=ayala $+2014+$ cisticercosis

51. Flisser A, Sarti E, Lightowlers M. Neurocysticercosis: regional status epidemiology, impact and control measures in the Americans. Acta Tropical.2003;8(1):43-51.Disponible en: http://www.ncbi.nlm.nih.gov/pubmed/?ter$\mathrm{m}=$ flisser $+2003+$ Neurocysticercosis

52. Bern CG. Magnitude of the disease burden from neurocysticercosis in a developing. Clinin. Infect. Dis.1999;29:12031209. Disponible en: http://www.ncbi.nlm.nih.gov/ pubmed/?term=Magnitude + of + the + disease + burden + from + neurocysticercosis + in + a+ developing

53. Nogales J, Arriagada C. Neurocisticercosis como paradigma de las parasitosis del sistema nervioso central. En J. Nogales, A. Donoso, \& R. Verdugo, Tratado de Neurología Clínica.2005;367-370. Santiago de Chile: Universitaria

54. Botero D, Restrepo M. Parasitosis Humana: Incluye animales venenosos y ponzoñosos. 5 Edicion. Medellin: CIB.2012:112-118.

55. Kater`ina P, Miroslav O, Ales` H, Kla'ra J', Norman G, Bruno L, Angelique T, Martin M, John K, David M. Novel Insights into the Genetic Diversity of Balantidium and Balantidium-like Cyst-forming Ciliates. J PLoS Negl Trop Dis.2013;7(3):1-10. Disponible en: https:// www.ncbi.nlm.nih.gov/pmc/articles/PMC3610628/ Doi: $10.1371 /$ journal.pntd.0002140

56. Encuesta Nacional de Parasitismo 2012-2014. Ministerio de Salud y Protección Social.2016. Disponible en : https://www.minsalud.gov.co/sites/rid/Lists/Bibliotec- 
aDigital/RIDE/VS/ED/GCFI/Herney\%20Rengifo.pdf

57. Gleason N, Kornblum R, Walzer P. Mesocestoides (cestoda) in a child in New Jersey treated with niclosamide (Yomesan). Am J Trop Med Hyg.1973;(6):757-60. Disponible en: http://www.ncbi.nlm.nih.gov/pubmed/4745235

58. Aguirre C, Angles R, Ash L. Coma S. Balantidiosis in Aymara children from the northern Bolivian Altiplano. Am J Trop Med Hyg. 1998;59:922-27

59. Koneman E. Diagnóstico Microbiológico. 3ra ed. Editorial Médica Panamericana.1998;721-23. Disponible en: http://vitae.ucv.ve/index_pdf.php?module=articulo_pd$\mathrm{f} \& \mathrm{n}=4767 \& \mathrm{rv}=107$

60. Giacometti A, Cirioni O, Balducci M, Drenaggi D, Quarta M. De Federicis M, Ruggeri P, Colapinto D, Ripani G, Scalise G. Epidemiologic features of intestinal parasit- ic infections in Italian mental institutions. Eur J Epidemiol.1997;13:825-30. Disponible en: https://www.jstor. org/stable/3581880?seq=1\#page_scan_tab_contents

61. Guzman R, Nessi P, Gonzalez H. Balantidium spp en cerdos y sus criadores: Prevalencia en comunidades de dos Estados de Venezuela. Academia Biomédica Digital.2013;54:1-10 Disponible en: http://vitae.ucv.ve/index_pdf.php?module $=$ articulo_pdf $\& n=4767 \& \mathrm{rv}=107$

62. Rey L. Parasitología. Parásitos o doenças parasitarias do Homen nas Américas e na África. 2da ed. Editorial Guanabara Koogan.2001; p. 397-399. Disponible en: http://bases.bireme.br/cgibin/wxislind.exe/ iah/online/? IsisScript=iah/iah.xis\&src=google\&base $=$ LILACS\&lang $=$ \&\&nextAction $=$ lnk\&exprSearch $=407068 \&$ indexSearch $=$ ID 